\title{
Technological Environmentality: Conceptualizing Technology as a Mediating Milieu
}

\author{
Ciano Aydin 1,2 - Margoth González Woge ${ }^{1}$. \\ Peter-Paul Verbeek ${ }^{1,3}$ (iD
}

Received: 9 May 2017 / Accepted: 8 March 2018 / Published online: 10 April 2018

(C) The Author(s) 2018

\begin{abstract}
After several technological revolutions in which technologies became ever more present in our daily lives, the digital technologies that are currently being developed are actually fading away from sight. Information and Communication Technologies (ICTs) are not only embedded in devices that we explicitly "use" but increasingly become an intrinsic part of the material environment in which we live. How to conceptualize the role of these new technological environments in human existence? And how to anticipate the ways in which these technologies will mediate our everyday lives? In order to answer these questions, we draw on two approaches that each offers a framework to conceptualize these new technological environments: Postphenomenology and Material Engagement Theory. As we will show, each on their own, these approaches fail to do justice to the new environmental role of technology and its implications for human existence. But by bringing together Postphenomenology's account of technological mediation and Material Engagement Theory's account of engaging with environments, it becomes possible to sufficiently account for the new environmental workings of technology. To do justice to these new workings of environmental technologies, we introduce and develop the concept of "Technological Environmentality."
\end{abstract}

Keywords Active technological environments · Postphenomenology $\cdot$ Material Engagement Theory $\cdot$ Technological mediation $\cdot$ Human-technology relations

Ciano Aydin, Margoth González Woge, and Peter-Paul Verbeek contributed equally to this article.

Peter-Paul Verbeek

p.p.c.c.verbeek@utwente.nl

1 Department of Philosophy, University of Twente, Enschede, Netherlands

2 Thomas More Professor of Philosophy, Delft University, Delft, Netherlands

3 Department of Techno-Anthropology, Aalborg University, Aalborg, Denmark 


\section{Introduction}

As the digital revolution keeps advancing, the technological texture of our world is rapidly changing. After several technological revolutions in which technologies became ever more present in our daily lives, the digital technologies that are currently being developed are actually fading away from sight. Information and Communication Technologies (ICTs) are not only embedded in devices that we explicitly "use" but increasingly become an intrinsic part of the material environment in which we live. Technological developments like Ambient Intelligence (AmI) and the Internet of Things (IoT) are giving rise to Smart Environments (SmEs) that equip our material world with sensors and communication networks that not only detect and perceive us but also work on us. ${ }^{1}$

Although a great part of the network that constitutes the current computer era is already invisible to us (wires, base stations, servers, antennas, satellites, etc.), in the upcoming years, the "computer as we know it" is expected to play a decreasing role in our lives. A new generation of technologies will move from our desktops and pockets to our environment, merging into all kinds of objects and material infrastructures (Weiser 1991; Bibri 2015). With this developing digital ecology, the technological transformation of our material culture is reaching a new level: we seem to be heading towards a new phase in the history of homo faber, in which its bio-artifactual nature is intimately being shaped by a new wave of powerful but concealed technologies.

In this paper, we propose to characterize this new type of environmental technologies as Active Technological Environments (ATEs): they are not just a mute and stable background for human existence, but they are actively involved with the human beings and material objects for whom and which they form an environment. These ATEs, as we will argue, give rise to the coming about of what we will call Technological Environmentality (TE), as a characteristic of human existence in a world of ATEs. This concept of TE highlights that the environment in which human existence plays itself out has taken on a technological character and that this environment is actively doing something, resulting in a new, technological condition for the life of homo faber.

How to conceptualize the role of these new technological environments in human existence? How to understand the ways in which these technologies will mediate our everyday lives and might even give rise to a next episode in the homo faber's process of self-transformation? In order to answer these questions, we will bring together two approaches that each offer a framework to conceptualize elements of the relations between humans and ATEs: Postphenomenology and Material Engagement Theory. As we will show, each on their own, these approaches fall short to do justice to the new environmental role of technology and its implications for human existence. But by expanding and integrating them, it becomes possible to sufficiently explain and conceptualize the new environmental workings of technology and their implications for TE.

\footnotetext{
${ }^{1}$ There are several notions that closely resemble AmI and IoT vision but that may have a slightly different focus, such as ubiquitous computing, pervasive computing, calm computing, wearable computing, invisible computing, affective computing, Things that Think, and everywhere computing. Most important is that they all belong to a wave in ICTs that aims to incorporate computer technology and artificial intelligence in everyday activities, objects, and environments.
} 
For both Postphenomenology and Material Engagement Theory, the environment has never been a neutral or passive feature in understanding the existential and cognitive complexity of human beings. Nevertheless, in both theories, its role has been overshadowed by the role of concrete objects and artifacts. Although their answers to the questions of "what things do" (Verbeek 2005) and "how things shape the mind" (Malafouris 2013) entail a broad consideration of material agency, their focus on things seems to be insufficient to tackle the question on the new kind of agency that arises from the merging of technologies with the environment. What kind of technological agency arises from the dynamic merging of technologies and our environment? In which sense do they enable, constrain, and regulate flow and interaction as interfaces to the environment? And to what extent are they becoming our environment themselves? Before discussing our two theoretical frameworks and proposing how they can strengthen one another, we will further elaborate what is new about ATEs.

\section{What Is "new" about Active Technological Environments?}

Increasingly complex sensors, system architectures, and software that can gather, store, manage, and analyze data with great sophistication give rise to a new dimension of human-technology relations: as technologies are aimed to become the environment itself, people will interact with it through voice, gestures, and cues like respiration and body temperature (Aarts and de Ruyter 2009). ICTs are ever more seamlessly and unobtrusively integrated in our lives, as ubiquitous "utilities" that will play a role similar to the one electricity is currently playing in our lives and that will reveal their functionality by sensing and predicting behavior, as well as adapting to and also influencing people.

The AmI and the IoT, as its standard platform, are two of the strongest paradigms that shape a vision of the future in which people are supported by SmEs. ${ }^{2}$ Still at an early stage of development, their full operability will depend on the accuracy of sensing technologies, the robustness of wireless communication, and the sophistication of the human-technology interaction models. By connecting homes and cities through a device mesh that is meant to be ubiquitous and always on, this vision seems to be preluding a network of fully mediated technological cocoons (Ihde 1990).

Smart Environments promise to improve different aspects of our daily livesranging from logistics and energy consumption to security and support. While liberating us from dull and mundane human tasks, they are also shaping human decisionmaking and giving rise to a new repertoire of experiences and behaviors, as well as cognitive capacities. Therefore, we need a broader understanding of the impact of these environments on human cognition and agency.

In contrast to computational and representational models of agency, we argue that what humans do and experience is best understood in relation to embodied interactions and engagements with their material culture and environment (Ihde 1990; Ingold

\footnotetext{
${ }^{2}$ As it can be observed in the European Information Technologies research agenda and the innovation strategies of most of the global Information and Technology companies (Van Est et al. 2016; Bibri 2015). According to the World Economic Forum, by 2022, more than one trillion networked sensors will be embedded in all kinds of objects as well as in the environment.
} 
2000; Malafouris 2013). The long-evolved trajectory of shelters and placemaking has shaped human agency and the experiential process of sense making in intimate (and mostly taken-for-granted) ways (Seamon 2015). Homes, offices, schools, parks, and cities have never been a neutral background in the process of human becoming: humans have created these supportive environments, but they have created humans too (Clark 2003). Within these bio-artifactual environments, humans have organized their everyday experiences, sustained their routines and bodily habits, as well as scaffolded their cognitive abilities (Aydin 2015). In this sense, many human capacities, cognitive and non-cognitive alike, have always depended on the fact that humans engineer their environment to support their activities (Laland et al. 2000; Godfrey-Smith 2014).

Such engineering processes have recently reached a new level as a digital texture is starting to envelop everyday objects in order to make the environment become smart. Imperceivable, user-sensitive, artificially intelligent electronics and software are aimed to merge seamlessly with our biological selves and "in so doing they will ultimately blur the boundary between the user and her knowledge-rich, responsive, unconsciously operating electronic environments" (Clark 2003: 34). ATEs can reconfigure human cognition, perception, and praxis in unprecedented ways, possibly even resulting in a new evolutionary dynamic, as embodied daily-life cultural practices have always shaped cognitive processes across the scales of time (Malafouris 2013).

In literature that focuses on the influence of artifacts on our cognitive processes, attempts are made to identify which condition technologies need to meet in order to substantially complement our cognitive processing or even become part of it. Often, these attempts build on, correct, or complement the conditions that were formulated by Clark and Chalmers (1998), namely constancy, facility, trust, and prior endorsement. Clowes (2015), focusing on so-called electronic memory (portable and wearable digital devises and the "cloud" of ever-present data functions and services), proposes four key properties of new technologies: totality, incorporability, autonomy, and entanglement. Although those key properties of new technologies meet Clark and Chalmer's four conditions, they are, according to him, not sufficient; it is necessary for them to meet two additional conditions: personalization and entrenchment (see also Sterelny (2010)) and epistemic possession. Clowes (2015) argues that resources made available by technologies that are not personalized to an individual's particular patterns of use and cannot be sufficiently possessed, i.e., are minimally cognitively penetrable, policable, and revisable, cannot be considered a proper part of an agent. However, even if the technology is not to count as an actual part of one's mind, it can, he argues, substantially enhance or diminish our cognitive processing.

Heersmink (2015) does not want to focus on the question of how technologies can become part of our cognitive processing but rather proposes a multidimensional framework to conceptualize the complementary integration between agents and artifacts. This framework is operationalized in a number of dimensions: information flow, reliability, durability, trust, procedural transparency, informational transparency, individualization, and transformation. These are not meant to provide a set of necessary and sufficient conditions, but to provide a toolbox for investigating the degree and nature of the integration of agent and artifact into "new systemic wholes." How information trajectories are established depends, says Heersmink (2015), on the material and functional organization of the different components of the integrated situated system. In addition, Heersmink (2015) points out that interacting with cognitive artifacts does 
not only transform the brain's representational properties but that it also transforms our embodied interactive skills.

ATEs are becoming a constant, invisible, and always-on background to a variety of everyday tasks that replace or augment our biological systems and/or our pre-existing bio-technological interactions (Floridi 2014). With Google Search, for example, it is often quicker and easier to find out facts we might otherwise recall using our biological memory. The increasingly ubiquitous computing technologies that enable us to have constant and reliable access to increasingly larger amounts of data are ever more competing with our organismic functions and resources in terms of scope of information, accessibility, and authority (Clowes 2015).

Wikipedia and Facebook most clearly highlight that ATEs are often entangled systems, the content of which is produced and maintained by the aggregated distributed activities of millions of people. Soliciting particular kinds of input from the human user community enables these technologies to perform tasks that no finite group of individuals would be able to accomplish. It is in this respect crucial to recognize that they do not merely collect and store data made available by humans. They also actively process the data in such a way that it can be reused and repurposed (Halpin et al. 2010). Google, for example, does not passively retain information, but its algorithms and processes that are used to find that information are also constantly updated and redirected in order to fulfill new functions. By connecting to and exchanging data with other devices and technologies, as expressed by the concept of IoT, ATEs are able to increasingly act autonomously. The extent to which ATEs will shape the course of their own cognitive development, will probably depend on their capacity and success to "self-structure their learning" (see Settles (2012) and Smart (2017)).

Smart (2017) stresses that the Internet has played a crucial role in creating the sort of environment in which a conventional symbol processing computational economy is able to display forms of intelligence that might have seemed utterly unattainable (see also Smart et al. (2017)). The Internet, as a form of cognitive ecology, enables personal information to be stored online and subjected to forms of online (e.g., cloud-based) manipulation and processing. It increasingly shapes, supports, sustains, and possibly even realizes our cognitive processing routines. The technological environment that influences the profile of our subsequent cognitive and epistemic endeavors is, as Smart (2017) points out, actively created and configured in a process of "online ecological engineering" or "online cognitive niche construction."

Although we still can see ourselves (both individually and collectively) as engaged in this engineering process, we must acknowledge that we are not the only "agents" that influence and can oversee its outcome. ATEs are becoming more invisible and transparent in use, but due to their relative autonomy and active processing nature, they, at the same time (and this is crucial), are becoming opaquer in their workings (Clowes 2015). The question to what extent we can influence the ecological engineering that creates TEs becomes relevant here. Clowes (2015) discusses MyLifeBits as an example that illustrates that is not impossible for a technology to be transparent-in-use but still have the right sort of cognitive penetrability qualities to allow epistemic possession. We believe that adequately identifying these normative questions requires a more comprehensive theoretical framework. 


\section{Postphenomenology}

The postphenomenological approach in philosophy of technology (Ihde 1990; Verbeek 2005; Rosenberger and Verbeek 2015) investigates technology in terms of its role in the relations between the human beings and the world. Building upon the phenomenological tradition, with its strong focus in the character and structure of the relations between humans and world, it investigates how technologies help to shape these relations. Rather than approaching technologies as objects in the material world that are used by human subjects, it sees them as part of the relations between humans and world. When we post a tweet, have an MRI scan made, or drive a car, we are not primarily focused on the technologies we use, but on the people we want to reach via Twitter, on our bodies that we are concerned about, or on the route we are taking to get somewhere. Technologies are not just part of our world, but mediate our relations with the world. Such technological mediations are never neutral, since they disclose the world in particular ways as they organize our routines and embodied habits, channel and resignify our social relations, and shape new ways of sense making as well as what we find ourselves capable of doing. ${ }^{3}$

Don Ihde has identified several ways in which technologies can play a role in human-world relations, ranging from being "embodied" (like a pair of glasses that we do not look at but through) and being "read" (like a thermometer that does not give a sensation but a number that represents the temperature) to being interacted with (like an ATM to take money from a bank account) and playing a role at the background of human experience (like the sound of an air conditioning system that is not always perceived as such but still shapes how people perceive their environment). In all of these relations, human intentionality is mediated by technologies in a different way: it can be directed at the world "through" a technology (like an "embodied" pair of glasses) or via a technological representation (like a thermometer that gives a number to indicate temperature), but it can also be directed at the technology itself (like an ATM that requires interaction with itself) or at the world with technologies as context (like the air conditioning system that switches on and off automatically).

\subsection{Background Relations in Postphenomenology}

In postphenomenological studies, the background relation with technology has received substantially less attention than the other human-technology relations (cf. Rosenberger and Verbeek 2015). ${ }^{4}$ We argue that the incorporation of computer technology and artificial intelligence in all kinds of objects and the environment calls for a further exploration of the structure and character of this background relation.

In Technology and the Lifeworld (1990), Ihde refers to background technologies as those that do not require foreground attention, since they become a kind of nearbackground environment themselves. Ihde exemplifies these relations with taskoriented machines and appliances that require explicit and repeated programming,

\footnotetext{
3 The Postphenomenology's philosophical approach is characterized by an empirical orientation that has brought about detailed descriptions of the human experience with technology. These descriptions draw from a nuanced typology that considers the embodied, hermeneutic, alterity, cyborg, immersion, and background character of technological mediations (Rosenberger and Verbeek 2015).

${ }^{4}$ For rare exceptions, see: Van den Eede (2011) and Kiran (2012).
} 
ranging from fridges to lighting, insulation, heating, and cooling systems. By acknowledging that they transform the gestalts of human experience, he does recognize that background technologies are anything but neutral. They actively shape our everyday experience in ways that are mostly taken for granted:

The machine activity in the role of background presence is not displaying either (...) a transparency or an opacity. The "withdrawal" of this technological function is phenomenologically distinct as a kind of "absence". The technology is, as it were, "to the side". Yet as an absent presence, it nevertheless becomes part of the experienced field of the inhabitant, a piece of the immediate environment (Ihde 1990).

According to Ihde (1990: 112), precisely because they are absent presences, background technologies "may exert more subtle indirect effects upon the way a world is experienced." Although their particular quality makes it difficult to pursue a contrastive existential analysis, highlighting their absent presence proves fruitful to investigate the technological texture of ATEs.

Within ATES, the sophistication of their problem-solving character entails that networked artificially intelligent devices will not only operate at the background of our attention and without any human intervention, but will also become active in new ways. For example, the repeated programming and engagement that technologies may once have required could now be achieved through the managerial skills of an AI home assistant. The functionality of (smart) objects would be delivered without any further interaction with their material affordances, as the necessity to meticulously manage and plan any task at hand would be replaced by exploiting regularities in the physical and social environment. In this sense, the bodily constitution of the household's lived geography, strongly shaped by daily life activities that are technologically mediated, will be reconfigured. As tasks will be solved and delivered while technologies smoothly run at the background of our attention, our experience and field of awareness will be importantly reorganized. Such reorganization relies on the transformation of our learned praxis of bodily habituation. As these bodily habits change, so does our immediate and intimate significance of our world, for this significance is in great part achieved through bodily routines. AI home assistants can impact what we feel capable of doing, what we think is worth our time, and also our dispositions towards solving problems. They will also foster new habits for everyday interaction, as we grow accustomed to their capacities through voice command, task relief, and cognitive aid.

\subsection{Expanding the Scope of Postphenomenology}

Connecting to Diane Michelfelder's (2015) analysis, there are two reasons to expand the current postphenomenological framework in order to be able to analyze ATEs adequately. Both reasons point to a necessary reconsideration of the role that "the world" is playing within Postphenomenology.

First of all, Postphenomenology focuses primarily on individual experience in relation to individual technologies, as the "I-technology-world" mediation structure makes clear. This individual understanding of human-technology relations works quite well with embodiment, hermeneutic, alterity, and cyborg types of mediation, because 
they typically involve individual technological artifacts, and because in these relations, the subject is intentionally directed at the world through the mediation of specific technologies (cf. Ihde 1990; Verbeek 2005). But as a new type of background technologies, ATEs are not merely "mediating" our agency and experience of the world: they are increasingly becoming the world themselves. As Active Technological Environments are meant to be hidden from everyday phenomenal experience, they do not mediate "between" humans and world but as part of our world.

A second reason to expand the postphenomenological framework is the fact that, in ATEs, mediation should not only be investigated from the perspective of an intentional human subject. We explicitly need to address the intentional role of the technological environment as well. Their mediating roles in human practices and perceptions are partly the result of the ways in which Active Technological Environments are "intentionally" directed at humans: they detect human beings, analyze them, and act on them. This implies that ATEs urge us to analyze as well how mediation works "from the world to the human."

Active Technological Environments, therefore, pose a new challenge to postphenomenological theory. As they operate "under the threshold of every-day phenomenological experience, as this experience is rooted in ordinary perception" (Michelfelder 2015: 243), they challenge our understanding of the relations and boundaries between humans and technologies. Their phenomenological "unavailability" cannot be equalled to the withdrawal from a user perspective (like a hammer that moves to the experiential background when people use it), nor to the background relations as initially described by Ihde (1990). ATEs up to an important degree are becoming part of the "world itself." The main challenges for the postphenomenological approach in relation to Active Technological Environments, hence, are to conceptualize the ways in which environmental technologies merge with our world and to approach the human-technology-world relation also from the side of the world and not only from the intentional human subject.

Precisely these two themes - the interactions between humans and environment, and the agency of this environment - have a central place in the approach of Material Engagement Theory (MET). In order to conceptualize the relations between humans and Active Technological Environments, therefore, we first need to investigate how the MET framework could contribute to this.

\section{Material Engagement Theory}

Just like Postphenomenology, MET theorizes the complex relations between human beings and their material environment. Though related to the phenomenological tradition, MET also has close connections to cultural anthropology and cognitive archeology. It has developed an embodied and situated approach to human and non-human agents, focusing on the hybrid and interactive relationships between embodied agents and material culture. In this approach, material artifacts do not function as mediators of human practices and perceptions, but rather take on a form of agency themselves, in our engagement with them. Moreover, unlike postphenomenology, MET is strongly focused on cognition. According to MET, material things "are capable of transforming and rearranging the structure of a cognitive task, either by reordering the steps of a task 
or by delegating part of a cognitive process to another agent (human or artifact). This process of extended reorganization (...) refers to an outward expansion of the cognitive system in order to forge extra-neural connections objectified through material culture, bodily action, and learning" (Malafouris 2013: 247).

Even though MET has not focused on the role of technology in material environments yet, it is worth exploring the possibilities to do so. As MET underlines that our long-evolved human constitution has always relied on material culture, not just as prostheses for our agency, but as an active part of our cognitive evolution (Clark 2003; Malafouris 2013), it subscribes to the main thesis of the extended mind, which states that cognitive processes are not bounded to the brain, but rather comprise extracorporeal non-biological devices. It is through the integration of such devices that we have been able to perform complex cognitive tasks and develop new repertoires of skills. In this sense, the boundaries between humans and technology are not blurred because of wires and implants are plugged into our "skin bag," but rather because of the strong plasticity of human cognition. Therefore, a better understanding of the implications of ATEs requires us to look at the mutual permeability, binding and structural coupling they may have with humans. Because of their "absent present" role, ATEs have deep implications for the future of homo faber, in Clark's (2003: 34) words, because "the most seamless of all integrations and the ones with the greatest potential to transform our lives and projects, are often precisely those that operate deep beneath the level of conscious awareness."

\subsection{Active Environments in the Material Engagement Theory}

MET brings materiality into the cognitive composition or fold by investigating the cognitive efficacy of past and present material culture. It explicitly opposes a cognitivist and computational account of things. Things are not to be understood in terms of symbolic representations (e.g., Margolis and Laurence 2007; Gardner 1985); they are not what language and the human mind make of them. A cognitivist perspective purifies human cognition and detaches it from the world. From such an approach, it remains unclear how representational structures can actually relate to the environment, and how they are enacted in real-life situations and in different cultural settings.

Instead of taking the mind-world dichotomy as a starting point, MET approaches things as an inseparable component of human thought. If human cognition, as Clark (1997: 98) proposes, "is fundamentally a means of engaging with the world," then material culture is consubstantial with mind. World and human cognition are ontologically inseparable. Take the classical example of the blind man's stick, as discussed by Bateson: "Where does the blind man's self begin? At the tip of the stick? At the handle of the stick? Or at some point half-way up the stick?" (1973: 318). It is simply impossible to draw a delimiting line across the extended cognitive system which determines the blind's man locomotion. This impossibility to draw a line is also expressed in the experience of the blind person himself: the blind man does not sense the stick, but rather the presence and absence of objects in the outside environment. The stick extends, as Merleau-Ponty describes (1962: 143), the scope and active radius of touch. Through time and practice, the blind man's stick is incorporated and made transparent. The blind man's stick provides a parallel to sight: if we would remove it, we would prevent him, one could say, from seeing. 
The cognitive efficacy of material culture lies primarily in the fact that it makes it possible for the mind to operate without the need of mental representation. Pens, paper, hands, and bodily senses are not simply tools actualized by an internal processor but the systemic and intrinsic components the interaction of which brings forth the cognitive process in question. Material engagement is, in the world of Malafouris, "the synergistic process by which, out of brains, bodies and things, mind emerges" (2004: 58).

The view that human cognition and world are inseparable has far reaching implications for the conceptualization of "agency" and "intentionality." For Malafouris (2008), pottery-making serves as the prototypical example to explain the material engagement approach to agency and intentionality. The dynamic coupling between the potter and the clay, Malafouris writes (2008: 24), looks like a "dance of agency"-using the words of Andrew Pickering (1995). The potter and the clay are equal partners in this dance; inside the loop of pottery-making it is impossible to separate cause from effect. The hand, the clay, and the wheel are in constant, permanent interaction from the beginning to the end of the process: "On the one hand, the centrifugal force imparted to the clay by the movement of the wheel and the hands of the potter; and on the other, the skillful guidance of this force by the potter's fingers, raising or pressing down the clay to the desired form" (2008: 34$)$. At one moment, the potter is consciously moving clay around and shaping it; at another moment, movement is happening to the potter and he becomes the extension of the material agent. Agency is a property or possession neither of humans nor of nonhumans. Agency is the relational and emergent product of material engagement.

From this perspective on agency, intentionality - which is often perceived as the major characteristic feature of agency — cannot be conceived as a fundamental property of human mental states to be "directed at, or about, or of objects and states of affairs in the world" (Searle 1983: 1). Agency is related to causal events in the physical world rather than to representational events in our mental world. Intentions cannot be separated from engagement with the world, as the concept of "prior intention" holds, which indicates an alleged intention to act before the act is actually taking place, as if there could be an "internal" representational state without it being connected to any pragmatic effect in the world. In the Material Engagement Theory, intentionality always involves "intention in action" (Malafouris 2008: 31), which is not an internal property but rather constitutes and is constituted both by humans and by things.

This primacy of action underlines the primacy of engagement: engagement always precedes intentionality. Before formulating an intention about a certain action or activity, we simply act and learn certain capabilities required for that action, like opening a door. Even when an intention to act is realized before the action actually takes place, and as such could be considered as a "prior intention" causing an intention in action, then this prior intention itself is already shaped by a "Background." Without the clay and its physical affordances, as the basic element of the potter's Background, the potter would not be able to form the intention to form the pot.

Against an internalist and computational approach to Background (see Searle (1983:143)), then, where the physical resources available in the background are simply used by the potter's body, following the command of the potter's brain, MET breaks down the division line between human intention and material affordance. The potter's brain, the muscles of the potter's body, the potter's sense organs, the affordances of the potter's wheel, the material properties of the clay, the morphological and typological 
prototypes of existing vessels as well as the general social context in which the activity takes place all together contribute to the formation of an intentional state. The "background" should in fact be seen as an extended intentional state (Malafouris 2008: 32).

From the perspective of MET, the background or "environment" is not a passive collection of different things that provide a context for human existence. Rather, it instigates and actualizes intentionality according to the "situational affordances" (Gibson 1979;Knappett 2005) of a given context of engagement: the specific possibilities for engagement and action that open up in specific environments. Affordances are relational properties in the environment that present possibilities for action. They are directly perceived by individuals instead of being mediated by mental representations like mental models (see also Greeno (1994)). Gibson's focus on the affordances of physical objects, such as doorknobs and chairs, fits well in METs highlighting of the importance of material culture.

In undermining an a priori hierarchy between brain, body, wheel, clay, vessels, and social context when it comes to the question of where cognitive processes reside, MET indeed brings materiality into the cognitive fold and shows how the material environment co-shapes our intentions and causes our actions. If the environment is the conditio sine qua non of intentionality, then intentionality can no longer be understood as an internal property that only can be attributed to humans, nor can it be used as the criterion for the attribution of agency to humans. The environment should be seen as an equal and active partner in the dance of agency and the coming about of intentionality. The concept of "background as environment" thus connects intentionality to the "extended mind thesis," which holds that the mind cannot be reduced to brain activity but extends itself beyond the boundaries of the skull and the skin (Clark and Chalmers 1998).

\subsection{Expanding the Scope of the Material Engagement Theory}

MET offers a rich analysis of the relations between human beings and their material environment, explaining how this environment helps to shape our cognitive capacities, our agency, and our intentionality. MET primarily focuses on low-tech or even traditional material environments, though. The prototypical examples and models used in MET are illustrative: a blind-man's cane, pottery-making, and shell beads from the Blombos Cave. The same can be said of the properties and features of these environments that are discussed in MET, also in relation to the concept of "affordances": wood, clay, vessels, doorknobs, etc. To what extent is it possible to expand these analysis to the growing class of present-day and emerging technological environments?

The MET's account and analyses of low-tech environments cannot simply be extrapolated to technological environments like the Internet of Things and Ambient Intelligence. The differences between both types of environments are not only quantitative but also qualitative: ATEs constitute a specific kind of technological environment that needs to be distinguished from the environmental role earlier artifacts or technologies have been playing. Characteristic for certain present-day and emerging high-tech environments, as we saw in Sect. 1, is the smart information and communication technologies that are ever more seamlessly incorporated in their texture. Although MET emphasizes the embodied and situated character of human agents, it is in its current form unable to recognize the smart and often hidden workings of and 
interconnections between the different parts of the environment and the meta-data that are generated and used by virtue of ICTs. As a consequence, it cannot account for the emergence of a new type of "environmental agency and intentionality." Understanding the dance of agency between humans and contemporary digital technological environment requires a specific understanding of the ways in which these technologies take part in this dance, with new ways of being "technologically-intentionally" directed at humans and being involved in the coming about of human intentions and actions.

Expanding its analysis to high-tech, seamlessly integrated systems are not an easy thing for MET, because its basic scope contains several "blind spots." First of all, as indicated earlier, MET is focused on low-tech and "basic" material environments. Its detailed description of the use of and engagement with individual artifacts and specific devices neglects the advance of interactive and interconnected technological environments that are able to anticipate human behavior. In addition, MET is primary concerned with the tangible materiality of the things that surround us (like clay, vessels, and doorknobs), whereas both the technological environments and their workings are often hidden from everyday phenomenal experience and out of reach of approaches concerned with experienceable materiality. They are smart and becoming smarter exactly, because they can increasingly function without requiring experience or attention. TEs are adapting and actively and seamlessly responding to us to such a degree that we, though they might be significant, are less noticing their workings. While growing into TEs, we will gradually also develop new skills that better correlate to TEs and make them ever more seamless. We are becoming part of a complex entanglement of technological structures that are fading from view.

Second, the MET's analysis tends to concentrate too much on the "user" of "things in the environment," disregarding the growing capacity of technological environments to use human agents for certain purposes. It is an illusion to think that we can control the ecology of invisible and active technologies, just as it is naïve to believe that we can control our natural ecology. This blind spot partly could be explained by the tendency of MET, being faithful to a phenomenological approach, to analyze things and their effects from a first-person perspective, whereas appreciating the distinctive, evergrowing capacity of technological environments to behave as "actants" requires a multitude of perspectives, both synchronic and diachronic.

MET recognizes that the environment is not a collection of passive things that the human mind uses and acts upon. However, the "activity" in TEs that MET appreciates is quite "weak" if we take into account the "extraordinary activity" we come across in certain current and emerging ATEs. Being "absently present," they confront us with a whole new range of questions and challenges. How can we expand the framework of MET to conceptualize this new material environment that humans engage with, in which interactive technologies have come to play a central role?

\section{Technological Environmentality}

We have now seen that both Postphenomenology and Material Engagement theory offer central concepts to analyze the relations between human beings and Active Technological Environments, but that they also have important shortcomings to conceptualize the specificities of currently emerging environmental technologies. 
Postphenomenology has a strong focus on human-technology relations, but ultimately fails to conceptualize the active role of technologies that merge with our environment and become our "world," while MET does conceptualize the active role of our material environment but fails to address technology in its own right, especially the specific forms of intentionality and agency implied in ATEs.

At the same time, it is exactly these two concepts of intentionality and agency that can bring the two together - agency being a central concept in MET and intentionality in Postphenomenology. In order to develop a closer understanding of Active Technological Environments, we will first discuss how the postphenomenological concept of "mediated agency" and the MET concept of the dance of agency can be connected to each other, in order to understand both the actions and interactions of humans and technological environments. Second, we will analyze what this expanded notion of agency implies for intentionality. We will bring together the MET notion of intentionality as resulting from the dance of agency between humans and environment with the notion of "technological intentionality" that plays a central role in Postphenomenology. Together, they can account for the specific ways in which human intentionality takes shape in relation to a new, technological environment that embodies specific forms of intentionality itself.

\subsection{Agency in Active Technological Environments}

In order to account for agency in ATEs, Postphenomenology and MET can augment each other in two distinct ways. First of all, we need to resolve the issue of how to conceptualize the agency of the material environment in relation to the concept of mediated agency: is agency to be located in things or in the relations between humans and things? And second, we need to bring the postphenomenological framework to address the specificities of technology as an environment in relation to the ways in which MET conceptualizes the agency of the material environment.

Let us first turn to the issue of "environmental agency" versus "mediated agency." As we saw, the postphenomenological approach does not attribute agency to material objects; it rather sees agency as the product of mediated relations between humans and world. In that sense, agency is "hybrid," containing both human and non-human elements. From the perspective of MET, this focus on technologically mediated agency does not go far enough. When analyzing how human beings deal with their material environment, this interaction needs to be seen as a form of mutual engagement. It is a dance of agency rather than "materially mediated human agency": a dance in which humans and environment engage with each other and in which the agency of the human and the agency of the material environment are closely intertwined, and together constitute a practice.

With regard to Technological Environments, then, the postphenomenological focus on mediation seemed to constitute a problem, because in technological environments this mediating role of technology - as part of the human-world relation-is less evident, since technologies in fact become invisible here and are not experienced themselves. Rather than being a mediator, technology in fact becomes part of our world here. For that reason, the MET approach, which thematizes the interplay of human and material agency in human cognition, offers a good alternative. MET does conceptualize the engaged interactions between human beings and their environment, 
but at the same time it requires a framework to understand the specificities of technological environments.

In order to solve this tension, it is important to see that the MET concept of "engagement" resulting in a dance of agency in fact embodies elements of mediation, while Postphenomenology does leave room to conceptualize a specific form of agency of the material environment. Upon closer inspection, two dimensions of agency play a role simultaneously when conceptualizing agency and the interactions between humans and their material environment. First, there is the interaction itself, which can be conceptualized as a "dance" in which human and material agency are interwoven and help to shape each other. But second, there is also a form of human agency resulting from this interaction. The interaction between potter and clay helps to shape the agency of the potter, not only in relation to the clay but also in relation to other people in the workshop and even results in a new material environment: pottery that organizes practices of eating, storing food, decorating, etc. The fact that the agency of human beings takes shape in close interaction with the material environment, then, can actually be seen as a form of mediation itself. In the dance of agency between humans and environment, the environment helps to shape human agency in a broader sense than only the agency in relation to that material environment itself.

In Active Technological Environments, this double dimension of agency is even more evident. Take the example of a Smart Environment like an elderly home that determines which doors will open and which will not, on the basis of RFID chips integrated in clothes, to prevent people with Alzheimer's disease to leave the house and lose their way, while still enabling them to move freely through the house. In this case, there is a clear interaction between the humans and the environment: a dance of agency between inhabitants of the house and the house itself. In this interaction, the agency of humans and of the environment are closely intertwined, resulting in a specific pattern of moving through the house, dealing with the house, and experiencing the house. But the implications of this material engagement reach far beyond the mere interaction between the inhabitants and the house itself: it also helps to shape how humans interact with each other, for instance, and how they do things in the house, rather than with it. As such, the smart environment here has a double agency: one that is part of the dance of agency between inhabitant and house and another one that mediates the relations between inhabitants and their world, like the other people they can meet, and the activities they can or cannot engage in.

Discussing this interaction between inhabitants and Active Technological Environments immediately makes visible the second way in which Postphenomenology and MET can come together. The technology in this example, after all, has a specific way of being directed at human beings: it embodies a specific technological intentionality, to phrase it in postphenomenological terms (Verbeek 2008). The sensor network in the house is directed at the RFID chips people are wearing and thus detects inhabitants in a highly specific way. Active Technological Environments have many other forms of technological intentionality available, like camera systems with pattern recognition algorithms that can recognize faces, or criminal activities, or sensor networks that can detect traffic congestion and occupancy of parking places, in order to redirect traffic.

This technological intentionality, in fact, is part of a form of environmental agency that seems to be missing in the postphenomenological focus on mediation. ATEs embody a form of agency, in being actively directed at human beings, and bringing 
in this directedness into the interaction between people and environment. Bringing together Postphenomenology and MET thus makes it possible to conceptualize both the interactions between human beings and ATEs and the ways in which ATEs mediate human practices and experiences. Living in ATEs shape human beings into specific subjects, because of the specific ways they "perceive" each other and work on each other, and because of the ways in which these environments do not only help to shape the people's interaction with the environment itself, but also with other people and things in their world, that this environment is part of.

\subsection{Environmental Intentionality: Technology as Mediating Milieu}

The concept of intentionality forms a second way to connect Postphenomenology and MET to each other and to Active Technological Environments. Again, Postphenomenology and MET seem to diverge here. While MET claims that intentionality is shaped in interactions between humans and the environment, for Postphenomenology intentionality is mediated by the technologies that human beings deal with. At the same time, as we saw in the previous section about agency, there are specific forms of technological intentionality that remain hidden in the MET approach, because it does not thematize the specific character of the technological agency (the mediation itself?) involved in the dance of agency between humans and environment.

Upon a closer look, though, the two approaches can actually augment each other in a productive way and are more in line with one another than they might seem to be at first sight. First of all, and following the argumentation about agency in the previous section, the MET approach to intentionality as emerging from the interplay between human and environmental agency is in fact closely related to the postphenomenological concept of mediated intentionality. In Postphenomenology, this technologically mediated intentionality has been explained with the help of the example of antenatal diagnostic technologies. Sonograms mediate the intentional relation between the expecting parent(s) and the fetus, constituting the fetus as a potential patient, as gendered, and as a human being that has a quasi-separate existence from the female body in which it is growing (Verbeek 2008). At the same time, it constitutes the expecting parent(s) as being responsible for the health condition of the fetus: conditions like Down's syndrome or Spina Bifida can be detected before birth, moving them from the realm of fate to the realm of human responsibility. This technologically mediated intentionality, then, also mediates human intentions to act, for instance in ethical decisions about abortion. From the perspective of MET, these technologically mediated intentionalities could be analyzed as the outcome of a subtle interplay between expecting parents and ultrasound scanner, from which intentionality emerges: the interaction between human beings and elements of their environment functions as a mediator of intentionality.

At the same time, though, the specific mediating role of Active Technological Environments has not been conceptualized in Postphenomenology, yet, for the reason that the mediating role of environmental technologies does not easily fit the humantechnology-world scheme that is at the heart of the postphenomenological approach: the technologically mediated intentional relations between humans and world. In order to analyze ATEs, then, we need to merge the postphenomenological concept of mediated intentionality with the METs' approach to environmentality and 
conceptualize the human-technology-world relations that play a role when technology mediates intentionality from an environmental role.

For doing this we can expand the concept of "immersion," that has been used before to characterize Smart Environments (Verbeek 2014, 219). With this concept, a specific configuration of humans and technologies can be indicated, in which technologies merge with our world, and at the same time have a bidirectional intentional relation with humans. Schematically, this configuration can be noted as

\section{immersion relation: human $\longleftrightarrow$ technology/world}

This relation has a fundamentally different character than the background relation, as thematized by Don Ihde. Ihde notes this relation as human (technology/world) (Ihde 1990). While both in the background relation and in the immersion relation technologies become our world themselves, the fundamental difference between both is the degree of interactivity between human beings and this environment. While background technologies primarily function as a context, immersion technologies are to be seen as interactive environments.

An essential element of the relation of immersion is the specific way in which technologies can be intentionally directed at human beings, as we saw in the example of elderly homes that was mentioned previously. Camera systems with face recognition software, websites that analyze browsing behavior and present personalized search results, sensor networks at airports that detect body temperature and patterns of movement - all of these technologies have a specific way of being directed at humans: they "interpret" humans on the basis of the way in which they perceive them. Moreover, this "perceptual" technological intentionality also forms a basis for technologies to act intentionally on human beings. Elderly homes, as we saw, can intentionally lock doors for specific people, just like some commercial websites intentionally present persuasive search results to make people buy more, and traffic systems direct cars in specific directions to avoid congestion. This technological intentionality, in the double meaning of perceptual and actional intentionality, needs to have a central place in a conceptualization of Technological Environmentality.

At the same time, though, the postphenomenological notion of immersion is missing a central element. By focusing only on the interactions between the humans and the immersive environment itself, it fails to conceptualize the implications of Active Technological Environments for human practices and experiences. This critique in fact mirrors the critique of the dance of agency concept we gave in the previous section. When finding themselves in an Active Technological Environment, human beings are not just interacting with that environment itself. Rather, this interactivity forms the basis for the way in which people act, perceive, and live their lives. Immersion technologies are not only interactive but mediating as well: they help to shape human practices and experiences, but in radically different ways than technologies that we "embody" (like a pair of glasses) or "read" (like a thermometer). This impact of Active Technological Environments is the central element of the way in which MET analyzes intentionality. For MET, intentionality is the result of the dance of agency between humans and environment, and, as we just saw, we can actually see this "emergent" intentionality as a form of mediated intentionality. From the interaction between Active Technological 
Environments and human beings, intentional relations emerge between human beings and their world.

Active Technological Environments can, therefore, still be considered as mediators, but in a way that radically differs from the other forms of mediation that have been conceptualized in Postphenomenology so far. The central insight underlying the postphenomenological concept of mediation is that we should not consider technologies as elements of the world, but of the relation between humans and world. In Active Technological Environments, though, technologies do become part of the world and still play a role as mediators.

Instead of being "media" that have a place "in the middle," as a "means" connecting humans and world, technologies become a "milieu" here. Interestingly, the words means, "medium," and milieu are all related to the concept of "being in the middle," being "in-between." Yet, the mediating role of a means - a technology that is actively used by human beings - is radically different than that of a "technological milieu," which is not actively used but which forms an interactive environment. While means play their mediating role by establishing a connection between user and environment, a milieu connects humans and world by interactively "encompassing" the ways in which humans are related to the world; like water is the milieu for fish, connecting them in specific ways to other fish and plants and objects and therefore helping to constitute them as fish and the environment as their world.

This, then, is what we would like to call Technological Environmentality. Technology is becoming a mediating milieu, merging with the world to the point of becoming invisible, but at the same time intentionally directed at humans and helping to shape how humans act, perceive, and live their lives. This radically new environmental character of human existence marks a new stage in the history of homo faber and, therefore, of the human condition itself.

Open Access This article is distributed under the terms of the Creative Commons Attribution 4.0 International License (http://creativecommons.org/licenses/by/4.0/), which permits unrestricted use, distribution, and reproduction in any medium, provided you give appropriate credit to the original author(s) and the source, provide a link to the Creative Commons license, and indicate if changes were made.

\section{References}

Aarts, E., \& de Ruyter, B. (2009). New research perspectives on ambient intelligence. Journal of Ambient Intelligence and Smart Environments, 1, 5-14.

Aydin, C. (2015). The artifactual mind: overcoming the 'Inside-Outside' dualism in the extended mind thesis and recognizing the technological dimension of cognition. Phenomenology and the Cognitive Sciences, 14(1), 73-94.

Bateson, G. (1973). Steps to an ecology of mind. London: Granada.

Bibri, S. E. (2015). The shaping of ambient intelligence and the Internet of Things. Paris: Atlantis Press.

Clark, A. (1997). Being there: putting brain, body, and world together again. Cambridge: MIT Press.

Clark, A. (2003). Natural born cyborgs: minds technologies and the future of human intelligence. New York: Oxford University Press.

Clark, A., \& Chalmers, D. (1998). The extended mind. Analysis, 58, 10-23.

Clowes, R. W. (2015). Thinking in the cloud: the cognitive incorporation of cloud-based technology. Philosophy and Technology, 28, 261-296.

Floridi, L. (2014). The fourth revolution: how the infosphere is reshaping human reality. Oxford: Oxford University Press.

Gardner, H. (1985). The mind's new science: a history of the cognitive revolution. New York: Basic Books.

Gibson, J. J. (1979). The ecological approach to visual perception. Boston: Houghton Mifflin. 
Godfrey-Smith, P. (2014). Philosophy of biology. Princeton: Princeton University Press.

Greeno, J. G. (1994). Gibson's affordances. Psychological Review, 101, 336-342.

Halpin, H., Clark, A., \& Wheeler, M. (2010). Towards a philosophy of the Web: representation, enaction, collective intelligence. In J. Halpin \& A. Monnin (Eds.), Philosophical engineering: toward a philosophy of the Web. Oxford: Wiley Blackwell.

Heersmink, R. (2015). Dimensions of integration in embedded and extended cognitive systems. Phenomenology and the Cognitive Sciences, 14(3), 577-598.

Ihde, D. (1990). Technology and the lifeworld: from garden to earth. Bloomington: Indiana University Press.

Ingold, T. (2000). The perception of the environment: essays on livelihood, dwelling and skill. London and New York: Routledge.

Kiran, A. (2012). Technological presence: actuality and potentiality in subject constitution. Human Studies, 35(1), 77-93.

Knappett, C. (2005). Thinking through material culture: an interdisciplinary perspective. Philadelphia: University of Pennsylvania Press.

Laland, K. N., Odling-Smee, J., \& Freedman, M. W. (2000). Niche construction, biological evolution and cultural change. Behavioral and Brain Sciences, 23, 131-146.

Malafouris, L. (2004). The cognitive basis of material engagement: where brain, body and culture conflate. In E. DeMarrais, C. Gosden, \& C. Renfrew (Eds.), Rethinking materiality: the engagement of mind with the material world. Cambridge: McDonald Institute for Archaeological Research.

Malafouris, L. (2008). At the potter's wheel: an argument for material agency. In C. Knappett \& L. Malafouris (Eds.), Material agency: towards a non-anthropocentric approach. New York: Springer.

Malafouris, L. (2013). How things shape the mind: a theory of material engagement. Cambridge: MIT Press.

Margolis, E., \& Laurence, S. (2007). The ontology of concepts - abstract objects or mental representations. Noûs, 41(4), 561-593.

Merleau-Ponty, M. (1962). Phenomenology of perception. London: Routledge.

Michelfelder, D. (2015). Postphenomenology with an eye to the future. In R. Rosenberger \& P. P. Verbeek (Eds.), Postphenomenological investigations: essays on human-technology relations (pp. 237-246). London: Lexington Books.

Pickering, A. (1995). The mangle of practice: time, agency, and science. Chicago: University of Chicago Press.

Rosenberger, R., \& Verbeek, P. P. (Eds.). (2015). Postphenomenological investigations: essays on humantechnology relations. London: Lexington Books.

Seamon, D. (2015). Situated cognition and the phenomenology of place: lifeworld, environmental embodiment, and immersion-in-world. Cognitive Processes, 1, 389-392.

Searle, J. R. (1983). Intentionality: an essay in the philosophy of mind. Cambridge: Cambridge University Press.

Settles, B. (2012). Active learning. Synthesis Lectures on Artificial Intelligence and Machine Learning, 6(1), $1-114$.

Smart, P. R. (2017). Situating machine intelligence within the cognitive ecology of the internet. Minds and Machines, 27(2), 357-380.

Smart, P. R., Heersmink, R., \& Clowes, R. W. (2017). The cognitive ecology of the Internet. In S. J. Cowley \& F. Vallée-Tourangeau (Eds.), Cognition beyond the brain (2nd ed., pp. 251-282). Dordrecht: Springer.

Sterelny, K. (2010). Minds: extended or scaffolded? Phenomenology and the Cognitive Sciences, 9(4), 465481 .

Van den Eede, Y. (2011). In between us: on the transparency and opacity of technological mediation. Foundations of Science, 16(2), 139-159.

Van Est R., et al. (2016). Rules for the digital human park. Two paradigmatic cases of breeding and taming human beings: human germline editing and persuasive technology. Background Paper for the 11th Global Summit of National Ethics/Bioethics Committees. Berlin.

Verbeek, P. P. (2005). What things do: philosophical reflections on technology, agency, and design. University Park: Pennsylvania State University Press.

Verbeek, P. P. (2008). Cyborg intentionality: rethinking the phenomenology of human-technology relations. Phenomenology and the Cognitive Sciences, 7(3), 387-395.

Verbeek, P. P. (2014). Designing the public sphere: information technologies and the politics of mediation. In L. Floridi (Ed.), The Onlife manifesto: being human in a hyperconnected era (pp. 217-227). Cham: Springer.

Weiser, M. (1991). The computer for the twenty-first century. Scientific American,94-104. 